\title{
Análise do Investimento Público em Educação no Brasil de 2000 até 2011: Uma Abordagem de Elasticidade-Renda
}

\author{
Public Investment Analysis in Brazil's Education Sector between 2000 and 2011: An \\ Income Elasticity Approach
}

Mateus Boldrine Abrita ${ }^{1}$

Artigo recebido para publicação em Ago/2013 e aceito para publicação em Set/2013.

\section{RESUMO}

A educação é fundamental para o desenvolvimento de um país, por isso esse estudo visa analisar a relação entre as receitas do Estado e os Gastos públicos em Educação no período de janeiro de 2000 até junho de 2011, utilizando o conceito da elasticidade-renda. Para mensurar essa relação é necessário estimar uma função econométrica e realizar uma Regressão Simples para posteriormente auferir a elasticidade-renda. Se o resultado for maior que uma unidade, os gastos em educação são elevados mais que proporcionalmente aos aumentos da renda do Governo, indicando que a área é prioridade para o país. Entretanto, se o resultado for menor que uma unidade, os gastos em educação são elevados menos que proporcionalmente aos aumentos da receita do Governo dando indícios que a área não é o foco principal. O resultado obtido foi de 0,77 demonstrando que os recursos são canalizados para a área da educação proporcionalmente aos aumentos da renda do governo, indicando que a área não é prioridade para o dispêndio dos recursos.

Palavras-chave: Educação. Investimento público e elasticidades.

\begin{abstract}
Education is fundamental to the development of a country, so this study objective to examine the relationship between state revenues, arising from society itself through taxes, and public spending on education, using the concept of income elasticity. To measure this relationship is necessary to estimate a function and perform an econometric regression simple to later earn the income elasticity. If the result is greater than one unit, spending on education is high more than proportionally to the increase of income of the government, indicating that the area is a priority for the country. However, if the result is less than one unit, spending on education are high less than proportionately to increases in revenue from the Government giving evidence that the area is not the main focus of the Government. The result obtained was 0.77 demonstrating that resources are channeled to the area of education in proportion to increases in government revenue, indicating that the area is not a priority for the expenditure of resources.
\end{abstract}

Keywords: Education. Public Investment and Elasticities.

\footnotetext{
${ }^{1}$ Graduação em ciências econômicas pela Universidade Federal de Mato Grosso do Sul (2010) e mestrado no Programa de Pós-Graduação em Ciências Econômicas pela Universidade Estadual de Maringá (2012). Professor efetivo na Universidade Estadual de Mato Grosso do Sul. E-mail: mateusabrita@uems.br
} 


\section{INTRODUÇÃO}

A educação possui um papel importante para o desenvolvimento da sociedade, sendo um direito garantido constitucionalmente a todos os brasileiros. Para Luz (2005) existe uma diferença considerável entre o que está expressamente garantido em papel, como na Constituição de 1988, e a efetiva concretização de determinado direito, nesse caso, o acesso à educação. Por esse motivo, o Estado possui um papel relevante, como legislador, articulador, provedor e fiscalizador da educação no país. Para que esse serviço seja prestado são necessários recursos, que geralmente são obtidos através dos tributos, como também uma boa gestão, porém essa análise foge ao escopo desse trabalho. Sendo o ponto de partida para o investimento em educação por parte do Estado a existência de recursos, é interessante uma análise sobre a situação do financiamento público educacional no Brasil.

Uma importante contribuição que o investimento em educação geralmente provoca nos países, argumenta Melchior (1997), é a significativa redução das desigualdades sociais. Quando existe uma rede educacional que proporciona igualdade de educação entre ricos e pobres, todas as faixas de renda da população podem alcançar diversas oportunidades de possuir maior renda, diminuindo assim a distância entre os padrões de vida mais elevados e mais baixos. Por esses motivos, fica evidente a importância da educação na sociedade e na consolidação de um país próspero e igualitário, contribuindo para o desenvolvimento da nação.

A arrecadação do governo brasileiro vem batendo recordes consecutivos nos últimos anos segundo dados da Secretaria do Tesouro Nacional (2010). As receitas federais totais, ou seja, a soma de vários tributos era de 116 bilhões no ano de 1997, passando para vultuosos 737 bilhões em 2009, um avanço de 635\%. Logo, é interessante verificar se esses ganhos de recursos advindos de uma maior arrecadação estão sendo canalizados para a área educacional e, ainda, analisar se esses repasses são proporcionalmente maiores ou menores em relação ao ganho absoluto, para isso, esse trabalho utilizará o conceito de elasticidade- renda.

Quando é desejado saber o grau de influência que alterações em determinada variável resultarão em termos de outra, o conceito de elasticidade é utilizado, "[...] Genericamente a elasticidade reflete o grau de reação ou sensibilidade de uma 
variável quando ocorrem alterações em outra variável" (VASCONCELLOS, 1998, p.48). Seguindo esse raciocínio, pode-se analisar o comportamento dos gastos do governo em educação em relação ao aumento da renda do governo, arrecadação, para que se possa ter uma melhor ideia de como a área educacional é valorizada pelo Estado.

Nesse sentido, o objetivo desse trabalho é auferir a elasticidade-renda dos gastos educacionais efetuados pelo setor público no Brasil, no período de janeiro de 2000 até junho de 2011, sendo a renda a arrecadação total do Estado e o bem, os gastos públicos em educação, representados pelo Fundo Nacional de Desenvolvimento da Educação (FNDE). Assim, pode-se ter uma melhor compreensão do quão importante é a área educacional para o governo, pois é interessante que maiores recursos devam ser, sempre que possível, disponibilizados para áreas tão relevantes. Para evidenciar essa importância, a elasticidade-renda pode ser enquadrada em três tipos: negativa, quando aumentos de renda levam a quedas no consumo, positiva e maior que 1 , aumentos de renda levam a aumentos mais que proporcionais no consumo e positiva menor que 1 , aumentos de renda levam a aumentos de consumo.

Para demonstrar que existe uma priorização da área educacional, o resultado da elasticidade-renda deve ser do tipo positiva maior que 1, pois assim indicará que, quando a arrecadação do governo aumenta, ou seja, a renda se eleva, os gastos ou consumo em educação tem um acréscimo mais que proporcional ao aumento inicial da renda, evidenciando assim o papel de destaque da área. Esse cálculo é feito com a utilização de um método quantitativo, a econometria, por meio de uma Regressão Simples, aos quais são auferidos os resultados que possibilitam determinar a elasticidade-renda. Assim, o artigo está dividido em quatro partes além dessa introdução, compondo consecutivamente uma abordagem teórica, os aspectos metodológicos, as análises de resultados e por fim uma as considerações finais. 


\section{FUNDAMENTAÇÃO TEÓRICA}

A importância da educação para uma sociedade e o desenvolvimento de um país é inegável. Freire (1995) argumentava que sem a alfabetização, por exemplo, não há possibilidade dos cidadãos exercerem plenamente seus direito e deveres, como gozar de direitos políticos e cívicos perfeitamente. Na teoria econômica, o crescimento de longo prazo é atingido perpassando pela educação, Blanchard (2007) afirma que o crescimento sustentado de uma economia deve-se ao progresso tecnológico resultante do estudo, pesquisa e desenvolvimento.

A respeito do direito a educação, Ranieri (2009) argumenta que ocorreu um avanço significativo após a Constituição Federal de 1988, no que diz respeito, ao sistema jurídico brasileiro, não apenas em relação às constituições anteriores, mas também avançando em garantias de outros direitos sociais. Assim, a autora defende que a educação é tanto um direito individual como direito coletivo, sendo muito importante para o Estado Democrático de Direito, pois a relação dessas duas dimensões forma um alicerce para promover a democracia, os direitos humanos e a proteção do meio ambiente, valores imprescindíveis da sociedade atual.

Outro importante ponto na relação entre o Estado e a educação está na Lei de Diretrizes e Bases da educação nacional (Lei no 9.394/96). A esse respeito, Vieira (2001) nos diz que como nem sempre as diretrizes constitucionais são devidamente consideradas e concretizadas, essa lei permite uma abundância de normas regulamentares, que impossibilita uma flexibilização das atividades educacionais.

Nesse sentido, Ranieri (2009) explica que a lei visa articular os diferentes níveis e sistemas de ensino, como a educação infantil, o ensino fundamental, médio e superior, e fixa que sob a coordenação da União, todos os entes políticos poderão atuar nos diferentes níveis de educação. Mas, para isso partem de uma regra geral, a educação infantil e fundamental será prioridade dos municípios, já os estados e Distrito Federal atuarão primordialmente no ensino fundamental e médio, considerando que o não oferecimento do ensino obrigatório pelo poder público ou sua oferta irregular, importará a responsabilidade da autoridade competente. Desse modo, para suportar esses encargos busca-se garantir recursos à manutenção e 
desenvolvimento do ensino, por isso o estudo do financiamento da educação é de fundamental importância.

Assim, esse trabalho é interessante, pois analisa a importância que o Estado está dando para uma área tão relevante como a educacional, verificando se os recursos advindos da arrecadação do governo, retirados da sociedade por meio de tributos, estão sendo canalizados para educação, que é sem dúvida um anseio da sociedade. Por meio do resultado da elasticidade-renda ficará evidenciado também se esse repasse de verba está tendo um acréscimo superior proporcionalmente ao aumento da arrecadação, o que indicaria que o Estado está priorizando a área. Portanto, é levantada a seguinte questão: Qual a elasticidade-renda do gasto público em educação no Brasil?

Diversos trabalhos abordam a problemática do investimento e financiamento público da educação. Especificamente na análise da elasticidade-renda do gasto em educação no Brasil, Moretto (1994) realizou um estudo abordando o tema para o período de 1965 a 1988, utilizando os gastos públicos na função educação e cultura como a variável consumo e o Produto Interno Bruto do país como a variável renda. Os resultados foram que os gastos em educação eram elevados mais que proporcionalmente ao aumento da renda, indicando que apesar da realidade da educação estar aquém do que muitos críticos pensariam ideal, os recursos estavam sendo repassados de acordo com a realidade da renda do Brasil naquele período. $\mathrm{O}$ índice obtido pela análise da elasticidade foi de 1,12 validando a conclusão da autora, mas outros estudos para um período mais recente indicam um cenário diferente.

Segundo Fígoli (2006), o Brasil está alcançado melhorias na educação. Está ocorrendo, nos últimos anos, uma redução da parcela da população na categoria "nenhuma educação formal", e o tempo de permanência na escola está aumentando. Entretanto, o país ainda tem um caminho para percorrer até que se atinjam índices com os patamares de países considerados desenvolvidos. Abrahão (2005) alerta que comparativamente aos demais países da América Latina e países da Organização para Cooperação e Desenvolvimento Econômico os gastos em educação, principalmente per capita, estão muito baixos, isso pode dar subsídios para a argumentação que a questão educacional não está na pauta principal da 
agenda política do Brasil, o que é muito preocupante dada à importância da área para o desenvolvimento do país e consolidação dos direitos e garantias cívicos.

No período de 1994 a 1999, Almeida (2001) conclui que ocorreu um esforço significativo em proporcionar uma elevação do gasto em educação para um patamar mais compatível com a realidade do Brasil. Porém, quando se olha para os números proporcionais ao indivíduo, gasto por aluno, por exemplo, os valores ainda são aquém do que se compatibiliza com uma educação de qualidade. Uma análise no período de 1995 a 2005 concluiu:

"[...] Por fim, deve-se pensar que parte razoável das necessidades educacionais da população brasileira ainda não foi atendida, o que vai exigir a ampliação do acesso a bens e serviços educacionais existentes e a ser criados. Além disso, é necessária a promoção de melhorias na qualidade da oferta de bens e serviços públicos prestados, o que também vai exigir a ampliação do gasto em dimensão ainda a ser estimada" (CASTRO, 2007, p. 874).

Pode-se observar que o Brasil fez algum esforço no sentido de aumentar os gastos em educação, entretanto, o que se verifica na realidade são volumes de recursos ainda muito abaixo de valores razoavelmente satisfatórios para que se atenda às necessidades da população. Uma das alternativas apontadas diz respeito à abertura do setor educacional ao grande capital privado internacional, mas esse caminho deve ser adotado com cautela. Oliveira (2009) faz uma análise da penetração dos grandes fundos financeiros no setor educacional do Brasil, principalmente no nível superior de ensino e alerta para os riscos que isso pode gerar, como a transformação do ensino em mercadoria e a oligopolização da oferta.

Para minimizar tais problemas o autor supracitado defende um resgate do setor público, dando ainda mais importância para os gastos governamentais em educação, resgate esse que, infelizmente, parece não estar presente no passado recente da realidade brasileira. A falta de prioridade em termos de gastos educacionais no Brasil pode ser observada em governos passados, como por exemplo, no período de 1995 a 2002, em que o Brasil gastou apenas 4\% do Produto Interno Bruto em ensino e $8 \%$ em juros e encargo da dívida pública, como aponta Pinto (2002). Isso evidencia que talvez a educação pública no país não esteja entre os principais elementos da agenda governamental, isso é preocupante, pois a 
educação pode minimizar mazelas, como a pobreza e reduzir desigualdades sociais, problemas que assolam nosso país.

Portanto, pode-se observar que ocorreram esforços por parte do Estado brasileiro, para que a educação angariasse mais recursos. Porém, a realidade demonstra que apesar da evolução, ainda falta muito para que a educação no país seja compatível com outros países que possuem renda até inferior a nossa.

\section{METODOLOGIA}

2.1 SÉRIES TEMPORAIS

\subsubsection{Definição}

A classe de fenômenos cujo processo observacional e consequente quantificação numérica geram uma sequência de dados distribuídos no tempo é denominada série temporal (SOUZA, 1989).

A natureza de uma série temporal e a estrutura de seu mecanismo gerador estão relacionadas com o intervalo de ocorrência das observações no tempo. Caso o levantamento das observações da série possa ser feito a qualquer momento do tempo, a série temporal é dita contínua, sendo denotada por $x(t)$ (GRANGER, 1977). Entretanto, de acordo com Granger (1977) e Nelson (1973), na maioria das séries, as observações são tomadas em intervalos de tempo discretos e equidistantes.

Uma série temporal discreta pode ser representada por $X^{T}=\left\{x_{1}, x_{2}, \ldots, x_{T}\right\}$, sendo que cada observação discreta $x_{t}$ está associada a um instante de tempo distinto, existindo uma relação de dependência serial entre essas observações (SOUZA, 1989).

\subsubsection{Objetivo}

Granger (1977) coloca como objetivo inicial da análise de séries temporais a realização de inferências sobre as propriedades ou características básicas do 
mecanismo gerador do processo estocástico das observações da série. Assim, através da abstração de regularidades contidas nos fenômenos observáveis de uma série temporal existe a possibilidade de se construir um modelo matemático como uma representação simplificada da realidade (BARBANCHO, 1970).

Após a formulação do modelo matemático, obtido pela seleção entre as alternativas de classes de modelos identificadas como apropriadas para essa representação e subsequente estimação de seus parâmetros, é possível utilizá-lo para testar alguma hipótese ou teoria a respeito do mecanismo gerador do processo estocástico e realizar a previsão de valores futuros da série temporal (NELSON, 1973).

\subsubsection{Previsão de Séries Temporais}

Segundo Barbancho (1970), uma previsão é uma manifestação relativa a sucessos desconhecidos em um futuro determinado. A previsão não constitui um fim em si, mas um meio de fornecer informações e subsídios para uma consequente tomada de decisão, visando atingir determinados objetivos (MORETTIN, 1981).

Considerando um conjunto de observações de uma série temporal coletadas até $o$ instante $t \mathrm{e}$ de um modelo que represente esses fenômenos, a previsão do valor da série no tempo $t+h$ (Figura 1) pode ser obtida.

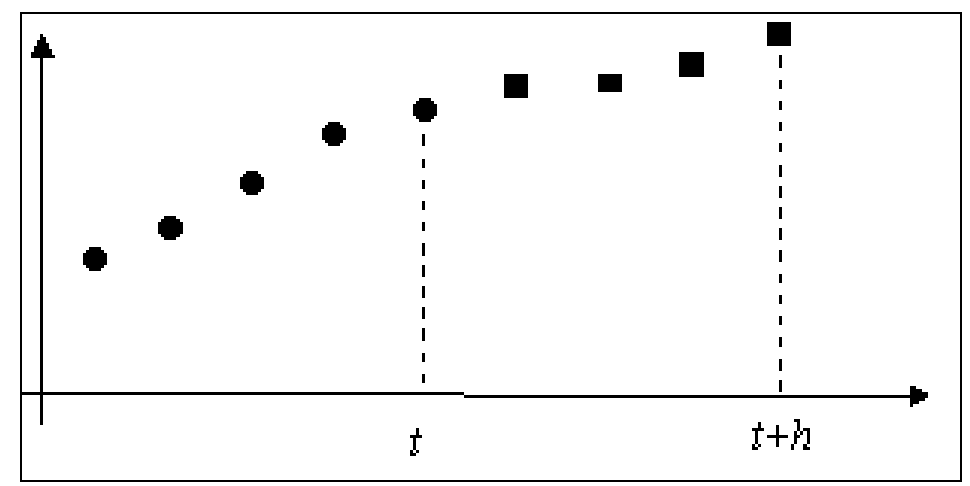

Figura 1: Observações de uma série temporal com previsões de origem $t$ e horizonte $h$ Fonte: GRANGER (1974). 
Dependendo do valor assumido pelo horizonte de previsão, Souza (1989) e Wheel Wrigth (1985) classificam as previsões dos valores futuros de uma série temporal como de curto, médio ou longo prazo. Desse modo, diante da possibilidade de existência de diferentes horizontes de previsão, Refenes (1993) especifica técnicas distintas para prognosticar os valores futuros de uma série temporal:

- previsão múltiplos passos: esta abordagem, adotada para longos horizontes de previsão, procura identificar as tendências gerais e os pontos de inflexão mais relevantes na série temporal. Na previsão múltiplos passos, o conjunto de valores correntes é empregado na realização da previsão para determinado instante; esta previsão é, então, introduzida entre as observações passadas, compondo, desta forma, um novo conjunto de dados, sobre o qual será obtida a previsão do tempo subsequente.

- previsão simples passo: nesta técnica não há incorporação de previsões aos dados utilizados para encontrar a próxima previsão, sendo esta, independente dos valores anteriormente previstos. A previsão é feita apenas para o período de tempo imediatamente posterior ao atual, a partir das observações da série temporal.

Para Souza (1989), a garantia da otimalidade das previsões de uma série temporal somente é alcançada adotando como horizonte de previsão o instante de tempo imediatamente subsequente à origem $t$.

Naturalmente, a investigação do poder preditivo do modelo especificado como o mais adequado para explicar o mecanismo gerador das observações de uma série temporal é um processo empírico de verificação, visto que são feitas comparações entre as previsões e as observações, a fim de confirmar a habilidade do modelo matemático em descrever a estrutura definida pelos dados da série temporal analisada (BARBANCHO, 1970).

\subsubsection{Métodos de Previsão de Séries Temporais}

Wheel Wrigth (1985) define um método de previsão como sendo o conjunto de procedimentos usados no desenvolvimento de uma determinada previsão. 
Os métodos de previsão de séries temporais, classificados como métodos quantitativos, baseiam suas previsões na extrapolação de características de observações passadas e no inter-relacionamento entre essas observações, fornecendo previsões acuradas se o futuro apresentar comportamento similar ao passado (WHEELWRIGHT, 1985).

De acordo com este autor, a maioria dos métodos de previsão de séries temporais se baseia na suposição de que observações passadas contêm todas as informações sobre o padrão de comportamento da série temporal e esse padrão é recorrente no tempo. O propósito dos métodos de previsão consiste em distinguir o padrão de qualquer ruído que possa estar contido nas observações e então usar esse padrão para prever os valores futuros da série temporal. Assim, pela identificação desse componente, a previsão para períodos de tempo subsequentes ao observado pode ser desenvolvida.

Apesar de quase a totalidade dos métodos de previsão de séries temporais estar fundamentada apenas na análise das observações da série de interesse para a especificação de algum modelo que descreva essas observações, alguns procedimentos de previsão tentam explicar o comportamento de uma série temporal pela evolução dos fenômenos observacionais de outras séries. Desta forma, dependendo do número de séries temporais envolvidas na modelagem, Souza (1989) classifica os métodos de previsão em univariados, funções de transferência e multivariados.

Os métodos univariados, que compreendem a maior parte dos métodos de previsão de séries temporais, consideram somente uma única série para a realização dos prognósticos. As previsões decorrentes da aplicação de métodos univariados podem estar relacionadas apenas com as informações contidas na série histórica de interesse (métodos baseados na estatística clássica) ou também, além de incorporarem essas informações, consideram outras supostamente relevantes e que não estão contidas na série analisada (métodos baseados na estatística bayesiana).

Aquelas metodologias nas quais a série de interesse é explicada não só pelo seu passado histórico, como também por outras séries temporais não-correlatas entre si, são conhecidas como funções de transferência. Esta classe de métodos de 
previsão envolve, portanto, mais de uma série temporal, com a ressalva de que a relação de causalidade entre estas séries é perfeitamente conhecida.

Os métodos multivariados abrangem os procedimentos de previsão que associam mais de uma série temporal na efetivação de prognósticos sem, no entanto, qualquer imposição com relação à causalidade entre essas séries.

Diante disso, há uma variedade enorme de métodos de previsão de séries temporais, cada qual com suas capacidades e limitações (WHEELWRIGTH, 1985). Qualquer que seja a classificação desses métodos é possível utilizar um número muito grande de métodos diferentes para descrever o comportamento de uma série particular. A seleção do método de previsão adequado depende de vários fatores, tais como o comportamento do fenômeno observável ou o conhecimento a priori que se tenha sobre a sua natureza e do objetivo da análise.

Souza (1989) e Wheel Wrigth (1985) relacionam os métodos de previsão baseados exclusivamente em uma única série histórica de dados em métodos de decomposição, métodos simples de previsão e métodos avançados de previsão de séries temporais.

\subsection{DADOS}

Neste estudo é utilizado como Proxy para os gastos públicos em educação o Fundo Nacional de Desenvolvimento da Educação (FNDE), disponibilizados pelo Ministério da Educação do Brasil. Esse fundo é constitucionalmente garantido e serve de fonte de recursos para o financiamento da educação no Brasil. A outra variável utilizada, como renda do governo, que são as receitas provenientes dos tributos, foram disponibilizadas pelo tesouro nacional do Brasil. O período de análise compreende janeiro de 2000 até junho de 2011, totalizando 138 observações, é importante destacar que o período de abrangência do estudo é escolhido a fim de incluir toda a década de 2000, entretanto, dificuldades de disponibilização de dados e periodização dos mesmos, também foram considerados na escolha do período. 


\section{ANÁLISE DOS RESULTADOS}

Os dados analisados neste trabalho foram retirados do Tesouro Nacional, do Ministério da Fazenda do Brasil, em valores de logarítimos (log).

São consideradas as variáveis de despesas com Fundo Nacional de Desenvolvimento da Educação (FNDE) (despesas) e o total de receitas tributárias arrecadadas pelo governo federal (receitas).

Após inserir estes dados no Stata, é necessário informar ao software, que estamos trabalhando com dados em séries temporais. Feito isto, é interessante visualizar graficamente como as variáveis em análise se comportam ao longo do tempo.

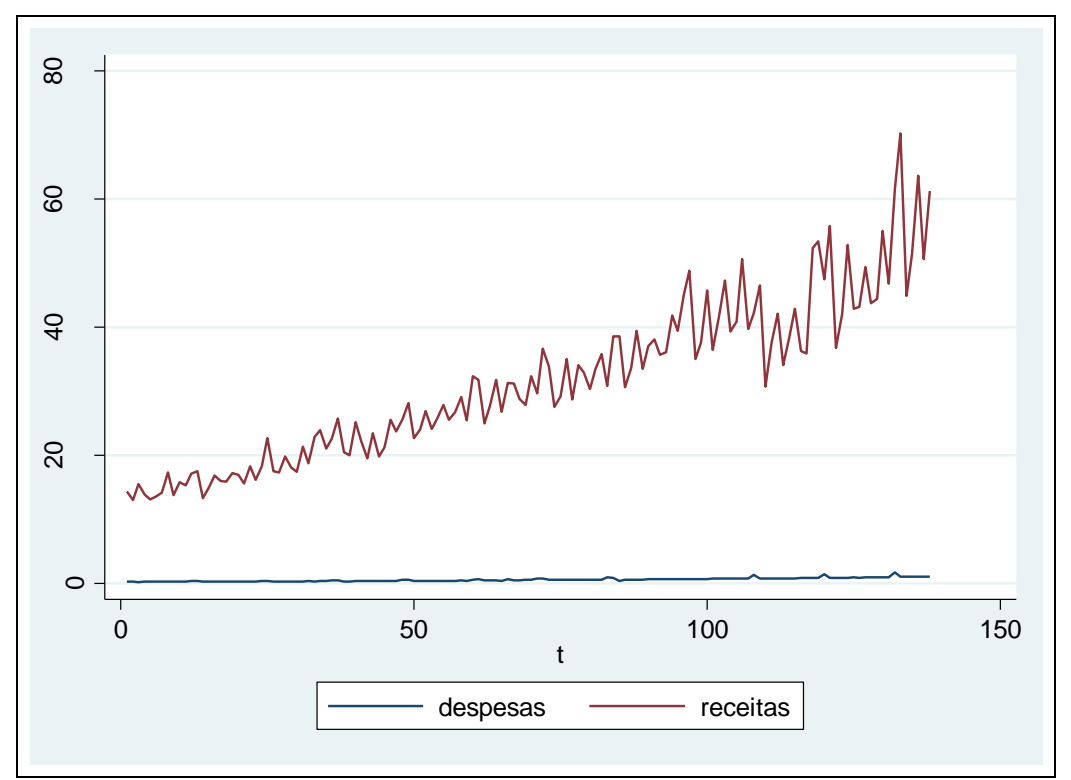

Gráfico 1: Comportamento das Receitas Tributárias (receitas) e Despesas com o Fundo Nacional de Desenvolvimento da Educação (despesas) ao longo do tempo.

Fonte: Elaboração própria por meio do software STATA e dados do Ministério da Fazenda do Brasil.

Ao observar o gráfico acima, é possível visualizar como estas variáveis se comportam ao longo tempo. Nota-se que as receitas têm um comportamento crescente, indicando que venha a ser uma série temporal não estacionária. Enquanto que a despesas mostrou-se estacionária ao longo do tempo.

Prosseguindo na análise do gráfico, no que se refere a variável receitas, pode haver uma tendência em dizer que estas séries são não estacionárias, pois visualmente, a média, a variância e a covariância destas séries parecem variar. 
Para verificar a estacionariedade das séries, será feito os testes de DickeyFuller aumentado e de Phillips-Perron, verificando se a série possui raiz unitária. Se a série possuir raiz unitária, ela é conhecida como série temporal de caminho aleatório, no entanto, se não tiver raiz unitária, a mesma vem a ser uma série temporal e é não estacionário.

Tabela 1: Teste Dickey-Fuller para Receitas Tributárias arrecadadas pelo governo federal (receitas).

\begin{tabular}{ccccc}
\hline & Estatística & $1 \%$ Crítico & $5 \%$ Crítico & $10 \%$ Crítico \\
\hline $\mathrm{Z}(\mathrm{t})$ & -2.572 & -3.498 & -2.888 & -2.578 \\
\hline
\end{tabular}

Aproximação $\mathrm{p}$-valor para $\mathrm{Z}(\mathrm{t})=0.0989$

Fonte: Elaboração própria por meio do software STATA e dados do Ministério da Fazenda do Brasil.

Pode ser observado que o valor absoluto do teste estatístico de DickeyFuller é menor do que os valores críticos a 1\%, 5\% e 10\%, assim, não aceitamos a hipótese nula do teste de que há raiz unitária, assim, a variável receitas é não estacionárias segundo o teste de Dickey-Fuller.

Tabela 2: Teste Dickey-Fuller para Despesas com Fundo Nacional de Desenvolvimento da Educação (FNDE) (despesas).

\begin{tabular}{lllll}
\hline & Estatística & $1 \%$ Crítico & $5 \%$ Crítico & $10 \%$ Crítico \\
\hline$Z(\mathrm{t})$ & -3.208 & -3.498 & -2.888 & -2.578
\end{tabular}

Aproximação $p$-valor para $Z(t)=0.0196$

Fonte: Elaboração própria por meio do software STATA e dados do Ministério da Fazenda do Brasil.

O segundo teste feito é o de Phillips-Perron, a forma de análise é semelhante ao teste de Dickey-Fuller.

Tabela 3: Teste Phillips-Perron para Receitas Tributárias arrecadadas pelo governo federal (receitas).

\begin{tabular}{ccccc}
\hline & Estatística & $1 \%$ Crítico & $5 \%$ Crítico & $10 \%$ Crítico \\
\hline$Z($ rho $)$ & -6.458 & -19.923 & -13.774 & -11.049 \\
$Z(t)$ & -1.618 & -3.498 & -2.888 & -2.578 \\
\hline
\end{tabular}

Aproximação $p$-valor para $Z(t)=0.4739$

Fonte: Elaboração própria por meio do software STATA e dados do Ministério da Fazenda do Brasil. 
Ambos os testes confirmam que as séries de receitas e despesas são nãoestacionárias.

Tabela 4: Teste Phillips-Perron para Despesas com Fundo Nacional de Desenvolvimento da Educação (FNDE) (despesas).

\begin{tabular}{lllll}
\hline & Estatística & $1 \%$ Crítico & $5 \%$ Crítico & $10 \%$ Crítico \\
\hline$Z($ rho $)$ & -11.710 & -19.923 & -13.774 & -11.049 \\
$Z(t)$ & -2.433 & -3.498 & -2.888 & -2.578
\end{tabular}

Aproximação $\mathrm{p}$-valor para $\mathrm{Z}(\mathrm{t})=0.1325$

Fonte: Elaboração própria por meio do software STATA e dados do Ministério da Fazenda do Brasil.

Posteriormente, é preciso verificar se a diferença é estacionária destas séries. Caso as mesmas forem, então temos uma série com ordem de integração igual a um ou I(1).

Tabela 5: Teste Dickey-Fuller primeira diferença para Receitas Tributárias arrecadadas pelo governo federal (receitas).

\begin{tabular}{ccccc}
\hline & Estatística & $1 \%$ Crítico & $5 \%$ Crítico & $10 \%$ Crítico \\
\hline $\mathrm{Z}(\mathrm{t})$ & -18.192 & -3.498 & -2.888 & -2.578 \\
\hline
\end{tabular}

Aproximação $p$-valor para $Z(t)=0.0000$

Fonte: Elaboração própria por meio do software STATA e dados do Ministério da Fazenda do Brasil.

Como podemos ver o teste de Dickey-Fuller aponta como estacionário a diferença e, portanto, indica que as séries são realmente I(1).

Tabela 6: Teste Dickey-Fuller primeira diferença para Despesas com Fundo Nacional de Desenvolvimento da Educação (FNDE) (despesas).

\begin{tabular}{lllll}
\hline & Estatística & $1 \%$ Crítico & $5 \%$ Crítico & $10 \%$ Crítico \\
\hline$Z(t)$ & -17.878 & -3.498 & -2.888 & -2.578 \\
\hline
\end{tabular}

Aproximação $\mathrm{p}$-valor para $Z(\mathrm{t})=0.0000$

Fonte: Elaboração própria por meio do software STATA e dados do Ministério da Fazenda do Brasil.

Assim, conforme os testes de Dickey-Fuller apresentados acima foram vistos que as variáveis receitas e despesas são PDE, pois as suas diferenças são estacionárias. 
Aplicando-se o Método dos Mínimos Quadrados à regressão da equação geral das receitas sobre as despesas do Fundo Nacional de Desenvolvimento da Educação (FNDE):

Ireceitas $=3,9304+0,77007$ ldespesas

Foram analisadas 138 observações para a estimação e os resultados encontrados foram:

$\mathrm{R}^{2}=0.8739 \quad \mathrm{R}^{2}$ aj $=0.8730 \quad \mathrm{RSS}=2.9334 \quad$ F-statistic $=942.86 \quad$ Durbin-Watson=2.001211 Nota: O R ${ }^{2}$ mede o grau de explicação da regressão; $R^{2}$ aj = $R^{2}$ ajustado; o F-statistic mede o nível de significância da associação entre as variáveis; RSS equivale à soma dos quadrados dos resíduos e Durbin-Watson é um teste que mede a presença de auto-correlação nos resíduos.

Fonte: Elaboração própria por meio do software STATA e dados do Ministério da Fazenda do Brasil.

Pelos dados acima, os resultados da regressão mostram-se satisfatórios. $\mathrm{O}$ coeficiente de determinação, $R^{2}$, assim como o valor de $F$, são altos. Também observou-se que a razão $t$ de "despesas" é positiva, na ordem de 0.77 , mostrando que o log a variável despesas do Fundo Nacional de Desenvolvimento da Educação (FNDE) tem uma boa relação, ou seja, despesas é positivamente relacionada a receitas. Se ldespesas for fixado em zero, o valor médio do Ireceitas é igual 3,9304. O efeito individual do aumento de uma unidade na variável Idespesas aumenta em 0,77007 o Ireceitas. E o teste de Durbin-Watson " $d$ " é significativo.

Granger e Newbold (1974) sugerem que um $R^{2}>d$ é uma boa regra prática para suspeitar que a regressão estimada sofre de regressão espúria. Porém, neste caso especificamente trabalhado, encontrou-se o contrário, ou seja, $d>R^{2}$.

Como se pode verificar, a um nível de determinação de $87 \%$, o valor final encontrado para o coeficiente de elasticidade das Despesas com Fundo Nacional de Desenvolvimento da Educação (FNDE) (Idespesas) no Brasil, no período de janeiro de 2000 a junho de 2011, foi de 0,77 , ou seja, menor que um $(<1)$. Isto quer dizer que a taxa de variação das Despesas com Fundo Nacional de Desenvolvimento da Educação (FNDE) é inferior à taxa de variação das Receitas Tributárias arrecadadas pelo governo federal. Ou ainda, os gastos com educação variam menos que proporcional, sendo tratada como bem normal e não como bem superior como deveria ser. 


\section{CONSIDERAÇÕES FINAIS}

Após os estudos realizados, conclui-se que o coeficiente de elasticidaderenda, indica que os recursos advindos dos tributos pagos pela sociedade brasileira estão sendo repassados para a área da educação apenas de forma proporcional. Assim, não está ocorrendo um acréscimo relativo dos valores, apenas um aumento nominal.

Esse resultado demonstra uma situação de piora ao ser comparado com o trabalho de Moretto (1994), cujo objetivo era abordar o tema para o período de 1965 a 1988. Naquela oportunidade, o autor concluiu que os gastos em educação eram elevados mais que proporcionalmente ao aumento da renda do governo, indicando que a área educacional estava tendo um ganho relativo ao longo dos anos em termo de recursos.

A situação atual, apesar de não ser totalmente ruim, mostra que a educação não está sendo considerada como prioridade por parte da sociedade e do governo brasileiro que a representa. Logo, uma elevação dos gastos em educação seria interessante, dada a relevância da área para o Estado Democrático de Direito, pois forma um alicerce para promover a democracia, os direitos humanos e a proteção do meio ambiente, auxiliando o crescimento econômico e o desenvolvimento nacional.

É sabido que apenas a elevação dos gastos propriamente dita não é condição suficiente para prover um ensino público de qualidade para todos os brasileiros, porém prover recursos suficientes para o financiamento da área é sim uma condição necessária para o bom funcionamento do setor. Também é importante salientar as limitações, principalmente de dados, que se apresentam em trabalhos dessa natureza, assim, é interessante que novos estudos sejam realizados com outras metodologias e diferentes bancos de dados com o propósito de melhor compreender a problemática do financiamento público da educação no Brasil.

\section{REFERÊNCIAS}

ABRAHAO, Jorge. Financiamento e gasto público da educação básica no Brasil e comparações com alguns países da OCDE e América Latina. Educ. Soc. 
[online]. 2005, vol.26, n.92, pp. 841-858. ISSN 0101-7330.

http://dx.doi.org/10.1590/S0101-73302005000300007.

ALMEIDA, Ivan Castro. Gastos com educação no período de 1994 a 1999. Revista Brasileira de Estudos Pedagógicos, Brasillia, v. 82, n. 200/201/202, jan./dez p. 137-198,. 2001

AMÉRICA LATINA. Educação e Sociedade, Campinas, vol. 26, n. 92, out, p. 841858, 2005.

BARBANCHO, Alfonso G. Fundamentos e Possibilidades da Econometria. Rio de Janeiro : Forum Editora, 1970. p. 18 - 32.

BLANCHARD, Oliver. Macroeconomia. Tradução Cláudia Martins, Mônica Rosemberg. 4ed. São Paulo: Pearson Prentice Hall, 2007.

CASTRO, Jorge Abrahão. FINANCIAMENTO E GASTO PÚBLICO NA EDUCAÇÃO BÁSICA NO BRASIL: 1995-2005. Educação e Sociedade, Campinas, vol. 28, n. 100 - Especial, out, p. 857-876, 2007.

FÍGOLI, Moema Gonçalves Bueno. Evolução da educação no Brasil: uma análise das taxas entre 1970 e 2000 segundo o grau da última série concluída. Revista Brasileira de Estudos de População, São Paulo, v. 23, n. 1, p. 129-150, jan./jun. 2006.

FREIRE, Paulo. Política e educação: ensaios. 2ed. São Paulo: Cortez, 1995.

GRANGER, C. W. J.; NEWBOLD, Paul. Forecasting Economic Time Series. New York : Academic Press, 1977.

GRANGER, Clive; NEWBOLD, Paul. Spurious regressions in econometrics. Journal of Econometrics, Nottingham, v.2, p. 111-120, Jul. 1974.

GUJARATI, D. N. Econometria Básica. Tradução Ernesto Yoshida. 3.ed. São Paulo: Pearson Prentice Hall, 2000.

LUZ, Liliene Xavier. Gestão, Financiamento e Controle Social da Educação. Teresina: EDUFPI, 2005.

MADDALA, G. S. Introdução à Econometria. Rio de Janeiro: LTC, 2001.

MELCHIOR, José Carlos de Araújo. Mudanças no financiamento da educação no Brasil. Campinas: Autores Associados, 1997.

MINISTÉRIO DA FAZENDA DO BRASIL. Dados sobre Arrecadação Bruta (Salário Educação). Fundo Nacional de Desenvolvimento da Educação (FNDE).

Disponível em: <http://www.fazenda.gov.br/>. Acesso em Dez. de 2011. 
MORETTIN, Pedro Alberto; TOLOI, Clélia Maria de Castro. Modelos para Previsão de Séries Temporais. In : $13^{\circ}$ Colóquio Brasileiro de Matemática. Rio de Janeiro : [s.n.], 1981.

NELSON, Charles R. Applied Time Series Analysis. San Francisco : Holden-Day, 1973.

OLIVEIRA, Romualdo Portela. A TRANSFORMAÇÃO DA EDUCAÇÃO EM MERCADORIA NO BRASIL. Educação e Sociedade, Campinas, v 30 ,n108, p 739760,out,2009.

PINTO, José Marcelino de Rezende. FINANCIAMENTO DA EDUCAÇÃO NO BRASIL: UM BALANÇO DO GOVERNO FHC (1995-2002). Educação e Sociedade, Campinas, vol. 23, n. 80, set, p. 108-135, 2002.

RANIERI, Nina Beatriz Stocco. Direito a Educação Aspectos Constitucionais. São Paulo: Editora da Universidade de São Paulo, 2009.

REFENES, A. N. et al. Financial Modelling using Neural Networks. London : [s.n.]. Department of Computer Sciences, University College London, 1993. p. 1 - 31.

SARTORIS, Alexandre. Estatística e introdução á economia. São Paulo : Saraiva, 2003 Secretaria do Tesouro Nacional. Disponível em <http://www.tesouro.fazenda.gov.br/estatistica/est_resultado.asp> acesso em 20 de julho/201

SOUZA, Reinaldo Castro. Modelos Estruturais para Previsão de Séries Temporais : Abordagens Clássica e Bayesiana. In : $17^{\circ}$ Colóquio Brasileiro de Matemática. Rio de Janeiro, 1989.

VASCONCELLOS, Marco Antonio Sandoval; GARCIA, Manuel Enriquez. Fundamentos de Economia. São Paulo: Saraiva, 1998.

VIEIRA, Evaldo. A poltiica e as bases do direito educacional. Cadernos CEDES, Campinas, V. 21, n 55,p. 09-29, Nov. 2001.

WHEELWRIGHT, Steven C.; MAKRIDAKIS, Spyros. Forecasting Methods for Management. 4th edition. New York : John Wiley \& Sons Inc, 1985. 
Revista Tecnologia e Sociedade - 2ª Edição, 2013.

ISSN (versão online): 1984-3526

ISSN (versão impressa): 1809-0044 